\title{
LA INFLUENCIA EXTERNA EN LAS POLÍTICAS EDUCATIVAS DE ARGENTINA, BRASIL Y CHILE (1990-2006)
}

\author{
A INFLUÊNCIA EXTERNA NAS POLÍTICAS EDUCATIVAS \\ DA ARGENTINA, BRASIL E CHILE (1990-2006)
}

THE EXTERNAL INFLUENCE IN THE EDUCATIONAL POLICIES OF ARGENTINA, BRAZIL AND CHILE (1990-2006)

L'INFLUENCE EXTERNE DANS LES POLITIQUES ÉDUCATIONNELLES DE L'ARGENTINE, LE BRÉSIL ET LE CHILI (1990-2006)

\author{
Xavier Rambla * \\ Antoni Verger ** \\ Aina Tarabini ***
}

\section{RESUMEN}

El objetivo del artículo es analizar el papel que juega la influencia internacional en el diseño $y$ aplicación de políticas educativas en el Cono Sur. Específicamente pretende explorar el proceso a través del cual las ideas, recomendaciones y metas globales modelan las políticas educativas latinoamericanas. Para ello, el artículo se centra en el programa de Educación para Todos y en el Acuerdo General de Comercio de Servicios, analizando los mecanismos de influencia externa que intervienen en cada caso -imposición, difusión y estandarización-, las formas a través de las cuales se "negocian" a escala nacional dichos programas y acuerdos y los efectos de dichos procesos en las políticas educativas nacionales de tres paises de la regiónArgentina, Brasil y Chile.

Palabras-clave: Globalización. Agenda educativa global. Educación para Todos. AGCS.

* Xavier Rambla, doctor en sociología (UAB, 1995) (Xavier.rambla@uab.cat).

** Antoni Verger, doctor en sociología (UAB, 2007) (A.Verger@uva.nl).

*** Aina Tarabini, doctora en sociología (UAB, 2008) (Aina.Tarabini@uab.cat).

Seminari d'Anàlisi de les Polítiques Socials (UAB) (http://sapsuab.wordpress.com/). Grup Interdisciplinari de Polítiques Educatives (UB, UAB) (http://www.ub.edu/gipe). Departament de Sociologia. Universitat Autònoma de Barcelona. Edifici B. Campus de Bellaterra. 08193 Bellaterra - Barcelona - Espanha. 
Este artículo es un producto del proyecto Más allá de la focalización: Educación, desarrollo y lucha contra la pobreza en el Cono Sur. Análisis de las aplicaciones de la nueva agenda política global en la región (MEC- España, SEJ2005- 04235). Es el primer análisis conjunto de las entrevistas y textos recopilados en varios trabajos de campo llevados a cabo en Santiago de Chile y Rancagua (2003, 2006), Belo Horizonte- Minas Gerais (2004, 2005), Buenos Aires, Salta, Córdoba, La Plata y Rosario (2004, 2006, 2008), Ginebra, París y Bruselas (2005, 2006, 2007). Agradecemos los comentarios recibidos en varias presentaciones anteriores de nuestras ideas en el Theorising Globalisation and Education Symposium (World Congress of Comparative Education Societies, Habana, 2004), en los Globalisation, Education and Societies Seminars (Univ. Bristol, 2005), en el Seminario Globalización y Desigualdad en América Latina (Centro de Investigaciones de Relaciones Internacionales y Desarrollo, Barcelona, 2005), en los Seminarios del Proyecto de Regulación de las Politicas Educativas (Univ. Buenos Aires, 2006), en las Amsterdam Institute of Metropolitan and Development Studies Lectures (Univ. Amsterdam, 2007), Seminario GIPE (UB- UAB, 2007), y en el Seminario Regulación de las políticas educativas en América Latina (Latin American Studies Association Conference, 2007).

Hoy en día es indiscutible que los organismos internacionales, los gobiernos más poderosos, los partidos, sindicatos y lobbies políticos, e incluso las empresas multinacionales, influyen en la política educativa de muchos gobiernos del mundo sino de todos ellos. La ejecución de iniciativas como el Programa Educación para Todos (concertado por Unesco con otras agencias), la inclusión de hitos educativos en los Objetivos de Desarrollo del Milenio (de Naciones Unidas), la negociación de acuerdos comerciales (como el Acuerdo General de Comercio de Servicios de la Organización Mundial del Comercio o varios Tratados de Libre Comercio) y los programas de cooperación internacional han abierto las puertas para que los ministerios de educación de todo el mundo reciban mensajes, recomendaciones, imposiciones y presiones a favor de unas determinadas medidas. De este modo, no sólo ha llegado a todos ellos el compromiso de alcanzar la escolaridad primaria universal hacia 2015 sino también toda una batería de orientaciones sobre la implicación de instituciones privadas en los sistemas educativos, la financiación de las escuelas, el uso de exámenes estandarizados, la movilidad de los estudiantes internacionales o la apertura de filiales universitarias a través de las fronteras.

En este artículo exploraremos el papel de esta influencia internacional en la aplicación del Programa Educación para Todos y del Acuerdo General de Comercio de Servicios en Argentina, Brasil y Chile. Se trata de tres países latinoamericanos que han asumido algunas condiciones y consejos de los organismos y donantes internacionales, pero han preservado un margen propio de maniobra consistente. Además, representan también una muestra de la región mundial donde los objetivos de la educación para todos han experimentado una variación relativa y positiva más acusada. Así, entre 1990 y 2000 la esperanza de vida escolar ha experimentado un avance medio mundial de +1.0 , 
mientras que el progreso latinoamericano ha alcanzado el valor de +2.6 (UNESCO, 2005: tabla 2.3).

En esencia, investigamos hasta qué punto dicha influencia ha transmitido las exigencias imperativas de los ajustes estructurales de los años ochenta y noventa, cuando se abrió el terreno global de la política educativa sometiendo a unos países endeudados a unos imperativos supuestamente ineludibles. Con ello, buscamos apoyos empíricos para una hipótesis sociológica, y a medio plazo esperamos sugerir argumentos sustanciales para extender la discusión democrática por encima de las fronteras de los estados.

\section{UN ANÁLISIS SOCIOLÓGICO DE LA AGENDA EDUCATIVA GLOBAL}

La globalización se plasma en varios síntomas de cambio social, como por ejemplo la formación de una agenda educativa global. Todas estas transformaciones forman una nueva madeja de interacciones sociales -estrechas, densas y multicausales- entre agentes sociales que no se hallan en una posición contigua. Se entablan relaciones, de cariz muy diverso, entre los emigrantes y sus familias de origen, entre los directivos empresariales y sus empleados off-shore, entre los movimientos sociales locales y globales, entre los turistas y los residentes en sus destinos vacacionales, entre las iglesias de varias religiones, entre los académicos de una especialidad empleados en las universidades de muchos países, o bien entre los defensores políticos y académicos de un ideal pedagógico propuesto simultáneamente en muchos sistemas escolares.

La agenda política es el repertorio de temas relevantes para la decisión de los gobiernos, compuesto de conceptos, herramientas de planificación, gestión y asignación, y de unos géneros apropiados del discurso político. Desde hace unos años varios especialistas han observado que la política educativa se ciñe a una agenda global (CARNOY, 1999; GREEN, 1999). Esta hipótesis no predice unas respuestas miméticas en todo el mundo, pero hace notar que todos los gobiernos, por activa y por pasiva, afrontan un mismo marco de acción política en educación, que delimita las prioridades, incide en las acciones concretas de los agentes educativos, interfiere en una variedad de luchas políticas y provoca muchas repercusiones distintas en cada sociedad.

El foco de análisis se dirige hacia las ideas políticas, que expresan las pretensiones y creencias de estos agentes. Las ideas separan lo preocupante de lo ordinario, o bien lo factible de lo inviable, abren y cierran puertas a unas y otras eventuales acciones efectivas, y promueven varias connotaciones ideológicas distintas. A nuestro entender, el análisis de la globalización educativa se debe inspirar en cuatro pistas sobre la génesis y la circulación de unas determinadas ideas por los circuitos locales, estatales y globales de la decisión política (HAY, 2002).

En primer lugar, una de estas claves estriba en las dimensiones del poder político, ya sea municipal, estatal o internacional. Weber y Simmel destacaron hace cien años que el 
poder consiste en la imposición vertical de unos intereses, órdenes y mandatos de unos agentes sociales dominantes a unos grupos subordinados. Pero los estudios políticos y lingüísticos más recientes han observado que el poder también consiste en la capacidad indirecta para moldear el contexto social donde se forman las preferencias y se toman las decisiones. Las ideas sobre las distintas políticas públicas, que unos agentes formulan en los ámbitos globales y otros recontextualizan en los estados, en las regiones y en las escuelas concretas, inspiran y legitiman las imposiciones al mismo tiempo que ejercen una persuasión mucho más sutil y polifacética en tanto en cuanto sugieren los temas importantes, catalogan los instrumentos de la acción institucional y modulan el margen de las acciones aceptables (HAY, 2002).

En segundo lugar, una trama de agentes sociales ha forjado esta faceta cognitiva de la globalización educativa. Muchos especialistas coinciden en subrayar que el Banco Mundial estrenó este espacio con sus ajustes estructurales de los años ochenta (BONAL, 2002). Simultáneamente, Unesco perdió su hegemonía a raíz de sus crisis internas y del reequilibrio de poder derivado de la ascensión de la Nueva Derecha en los países anglosajones (MUNDY; IGA, 2003). La Organización Mundial del Comercio, a su vez, nació en 1994 promoviendo unas reglas del juego económico que perfilan nuevos territorios educativos (ROBERTSON et al., 2002). Y la OCDE ha entrado en la cuestión con su discurso sobre la sociedad del conocimiento (RIZVI; LINGARD, 2006).

La tercera clave es la generalización entre las opiniones públicas de muchos países de unas creencias colectivas sobre cuáles pueden ser los resortes fundamentales de la expansión escolar y de sus consecuencias (DALE, 2005; PAWSON, 2002; REIMERS et al., 2006). Su núcleo intelectual asume que el libre comercio y la liberalización de las economías impulsan el desarrollo y alivian la pobreza (ROBERTSON et al., 2007), a lo cual se añade normalmente el consejo de establecer una gobernanza adecuada (WORLD BANK, 2004b). Por ejemplo, en el Cono Sur después de la "década perdida" las preocupaciones materiales se han suavizado y las recomendaciones sobre la gobernanza han arreciado. Como en todo el mundo, este recetario se apoya en varios recursos retóricos propios del registro científico, como las políticas basadas en la evidencia, las recomendaciones sobre la efectividad escolar y la consulta indiscriminada de indicadores estadísticos y de bancos de buenas prácticas para casi todos los temas (OZGA et al., 2006).

Por último, la cuarta guía de nuestro análisis es la hipótesis de Roger Dale (1999, $2000,2005)$ sobre la globalización de la política educativa. Según este autor, desde el siglo diecinueve los ministerios de educación han tomado prestadas unas medidas de unos y otros, y los pedagogos teóricos y prácticos han aprendido de otros países, pero hoy en día las mayores influencias llegan, más allá de estos canales bilaterales, por unos nuevos canales que han adquirido un verdadero estatus supra-estatal. Tres de ellos son especialmente significativos para nuestro análisis, a saber: la difusión, la estandarización y la imposición. 
- La difusión afecta a muchos campos de la política a un mismo tiempo, porque consiste en la extensión de un programa de acción mediante el discurso de los gobiernos (p. ej. la defensa de medidas de educación compensatoria y de liberalización de los servicios en Estados Unidos y la Unión Europea), de las cumbres regulares (p. ej., las conclusiones anuales del G-8) y de los organismos internacionales (p. ej. las publicaciones del Banco Mundial sobre la gobernanza o de la OCDE sobre la sociedad del conocimiento). Por tanto, afecta indirectamente a las decisiones gubernamentales en tanto en cuanto delimita las medidas más recomendables, en un extremo, y las opciones 'impensables', en otro.

- La estandarización incide en las reglas del juego político, por ejemplo constituyendo sistemas estadísticos integrados y comunes a muchos países (como los indicadores de Education at a Glance de la OCDE) o bien perfilando un tipo de leyes (como las estipulaciones del AGCS sobre el acceso al mercado de los servicios educativos). Aunque en cada país se pueden satisfacer estos criterios de distinto modo, su misma vigencia comporta que las acciones gubernamentales sean más visibles si hacen mella en los indicadores, o menos recomendables si encierran un mínimo riesgo de vulnerar un estándar internacional.

- Por último, la imposición exterior de una política educativa, normalmente activada mediante las condiciones asociadas a un crédito de una potencia extranjera o de una institución financiera internacional, pone en solfa tanto las decisiones como los márgenes de acción y las reglas del juego. Los gobiernos endeudados han decidido acogerse a un Plan de Ajuste Estructural, o aplicar los Poverty Reduction Strategic Papers del Banco Mundial en un momento dado. Estas decisiones dan por sentada una lista temas pertinentes y aplican reformas legales parecidas para alcanzar metas cuantitativas equiparables (véase Cuadro 1).

En sus interacciones bilaterales los gobiernos tomaban (y toman) una decisión explícita al hacer suya una política ajena, pero hoy en día les llegan constricciones mucho más sutiles a través de estos nuevos canales. Así, la difusión perfila unos marcos de la acción legitimada por los informes internacionales, las publicaciones aceptadas en las revistas académicas más prestigiosas o los bancos de buenas prácticas del Banco Mundial y de Unesco. A su vez, un gobierno hace suyas unas reglas del juego político que se han estandarizado en determinados foros y organismos internacionales cuando establece quién puede participar en un sector educativo (escuelas públicas, privadas, subvencionadas o pagadas, fundaciones, empresas, nacionales e internacionales), o bien cuando marca unos objetivos comunes de convergencia (mediante indicadores estadísticos, plazos para aprobar leyes, estatutos de unas determinadas instituciones, etc.). La imposición surte a un mismo tiempo todos estos efectos cuando un organismo internacional justifica qué tipo de programas sociales está dispuesto a sufragar (véase Cuadro 1). 
Cuadro I. Canales de la influencia exterior sobre la política educativa

\begin{tabular}{|c|c|c|c|c|}
\hline $\begin{array}{l}\text { Influencia } \\
\text { exterior mediante }\end{array}$ & $\begin{array}{l}\text { Los gobiernos } \\
\text { toman }\end{array}$ & $\begin{array}{l}\text { decisiones } \\
\text { conscientes }\end{array}$ & $\begin{array}{c}\text { dentro de unos } \\
\text { márgenes de acción }\end{array}$ & $\begin{array}{c}\text { según unas reglas } \\
\text { del juego }\end{array}$ \\
\hline \multicolumn{2}{|c|}{ Préstamo y aprendizaje } & XXXXXXXX & & \\
\hline \multicolumn{2}{|l|}{ Difusión } & & XXXXXXXX & \\
\hline \multicolumn{2}{|l|}{ Estandarización } & & & XXXXXXXX \\
\hline \multicolumn{2}{|l|}{ Imposición } & XXXXXXXX & XXXXXXXX & XXXXXXXX \\
\hline
\end{tabular}

Fuente: Elaboración propia a partir de Dale (1999).

En esencia, queremos averiguar si las ideas globales moldean las políticas educativas latinoamericanas. Nuestro balance provisional nos permite señalar tres pruebas iniciales que acreditan las hipótesis sobre las creencias colectivas (acerca de los beneficios de la educación) y sobre las formas implícitas de influencia externa. En primer lugar, las políticas educativas de estos países responden a un mismo paradigma científico- político; en segundo lugar, las recomendaciones del programa de Educación para Todos han llegado al Cono Sur principalmente a través de la estandarización de las transferencias y de la evaluación, así como de la difusión de la educación inclusiva; y finalmente, el AGCS ha estandarizado en esta zona, como en todo el mundo, un catálogo de servicios educativos y ha difundido la idea de la comercialización de dichos servicios. Los tres apartados siguientes exponen los pormenores de estos datos.

\section{EL PARADIGMA CIENTÍFICO- POLÍTICO DE LA AGENDA EDUCATIVA GLOBAL}

Ciertamente, la agenda educativa contiene una guía para la acción, un orden de temas según su prioridad y un abanico de instrumentos; sin embargo, todos los actores políticos apoyan sus intereses en juicios de hecho arropados por una supuesta validez científica. De esta manera, cualquier afirmación fáctica incluida en un informe oficial provoca un sinfín de reverberaciones, y cualquier opinión o propuesta concreta busca sistemáticamente unos avales de este tipo. Por tanto, no es ninguna sorpresa que el Programa Educación para Todos y el Acuerdo General de Comercio de Servicios hayan sido interpretados con estas pautas en Argentina, Brasil y Chile.

Los dos principales organismos internacionales implicados en la política educativa, la Unesco y el Banco Mundial, utilizan continuamente esta retórica híbrida que aglutina referencias políticas y científicas. Así, para Unesco el conjunto de América Latina ha adquirido una relevancia particular en los juicios sobre la gobernanza y el desarrollo de la educación para todos. En su opinión, el problema emblemático del continente, una 
vez consolidados varios avances cuantitativos, es afianzar una educación de calidad para todos (UNESCO- LLECE, 2002). Es muy llamativa la distancia entre sus alocuciones humanistas (p.ej. en el Informe Delors) y algunos argumentos dirigidos a los estados, en que coloca los beneficios económicos en el primer plano del impacto de esta educación de calidad sobre el desarrollo. Para ello recurre a una lista de afirmaciones categóricas sobre las correlaciones estadísticas entre la educación y sus esperados beneficios, que se convierten en alocuciones a favor de unas acciones determinadas.

The impact of education quality on development goals

- Impact of quality on individual incomes (...)

- Impact of quality on economic growth (...)

- Quality and non-cognitive skills (...)

- The impact of quality on behavioural change (...)

[gender equality, resilience in fighting AIDS] (UNESCO, 2005, p. 40-44).

De su parte, el Banco Mundial ha destacado varias oportunidades por las que la EPT puede favorecer la eficiencia institucional, interpretando de nuevo unas conexiones empíricas como la base de las recomendaciones pragmáticas (WORLD BANK, 2004a, p. 1). En América Latina, además, el Banco espera que la educación de calidad rompa varios resortes de la crónica desigualdad regional. Las medidas prometedoras en este sentido son el creciente acceso y la eventual articulación de medidas selectivas a favor de los pobres con los pilares universales del estado del bienestar. Este plan de actuación también confía en extrapolar tendencias empíricas detectadas en los informes de diagnóstico.

Latin America's inequality has deep historical roots and pervades contemporary institutions. But a "break with history" is possible through a range of economic policy levers.

Broadening asset ownership: redistribution with growth

- Democratizing education (...)

Developing deeper markets and more equitable institutions (...)

Avoiding crises and regressive workouts from macroeconomic shocks

- (...) Coupling conditional cash transfers to the poor with overall expansions of basic education and health services makes eminent sense both from the point of view of efficiency (since otherwise the poor may not demand these services) and the political sustainability of targeted programs (WORLD BANK, 2004b, p. 7-21).

En cuanto al AGCS, sus defensores esgrimen una serie de ventajas económicas e institucionales que el libre comercio educativo debe aportar al funcionamiento de los sistemas educativos y, a través de ellos, a la competitividad y la cohesión social de los signatarios del tratado. La misma idea de la comercialización de los servicios se ha extendido desde una "escuela" académica hasta los gobiernos a través de la labor de investigadores como Geza Feketekuty (2005). Varios especialistas argumentan que este 
acuerdo puede favorecer el desarrollo humano porque en el preámbulo del mismo AGCS se reconoce el "derecho de los miembros a reglamentar el suministro de servicios en su territorio y a establecer nuevas reglamentaciones al respecto, con la finalidad de realizar los objetivos de su política nacional, y la especial necesidad de los países en desarrollo de ejercer este derecho, dadas las asimetrías existentes en cuanto al grado de de desarrollo de las reglamentaciones sobre servicios los distintos países" (SAUVÉ, 2002). Moviéndose dentro de estos parámetros, el gobierno y las universidades chilenas han creado el Comité Exportador de Servicios Universitarios para contribuir al desarrollo del país vendiendo sus atractivos en el mercado mundial, para captar estudiantes extranjeros y para abrir sus propias universidades al mundo (VERGER, 2009).

En suma, el reflejo latinoamericano de la agenda educativa local ha cuajado en dos ideas: de una parte, el programa EPT puede "hacer que los servicios (educativos) funcionen para los pobres", de otra, el AGCS puede ensanchar la oferta de servicios educativos. Ambos argumentos comparten un mismo marco de referencia con los ajustes estructurales impuestos hace un par de décadas. En la medida en que este marco delimita los problemas prioritarios y las herramientas convencionales para resolverlos, configura algo parecido a lo que los filósofos de la ciencia llamarían un "paradigma políticocientífico" (si se nos permite la analogía), que conecta las políticas educativas con la lucha contra la pobreza.

\section{LA DIFUSIÓNY LA ESTANDARIZACIÓN DE LA EDUCACIÓN PARA TODOS EN EL CONO SUR}

El cuadro 2 esboza los canales de la influencia supra-global sobre la aplicación del Programa Educación para Todos en Argentina, Brasil y Chile. Básicamente, estos canales han sido la estandarización de las fórmulas de financiación y de los sistemas de evaluación, por un lado, y la difusión de ideas sobre la "focalización educativa", por otro (TARABINI, 2007).

La Educación para Todos ha marcado unas reglas normalizadas o estandarizadas de la financiación educativa y de la evaluación institucional de las escuelas. En ambos terrenos el Banco Mundial y el Banco Interamericano de Desarrollo han promovido buenas prácticas para asegurar que los recursos públicos llegan a los sectores sociales más necesitados. En Brasil, el país más pobre de los tres, se ha experimentado un mecanismo de financiación municipal que condiciona el presupuesto local a la extensión de la educación primaria. Esta fórmula se probó en este caso con la intención de convertirla en una buena práctica recomendada a todos los países menos desarrollados. 
Cuadro 2. Influencia externa sobre la Educación para Todos en Argentina, Brasil y Chile: estandarización y difusión

\begin{tabular}{|c|c|c|}
\hline $\begin{array}{l}\text { Objetivos de la Ed. } \\
\text { para Todos }\end{array}$ & $\begin{array}{l}\text { Alcanzar la escolarización } \\
\text { neta universal en primaria }\end{array}$ & $\begin{array}{l}\text { Garantizar una educación de } \\
\text { calidad para todos }\end{array}$ \\
\hline \multicolumn{3}{|c|}{ Estandarización } \\
\hline Argentina & $\begin{array}{c}\text { Plan Jefas/es de Hogar y } \\
\text { Programa Familias para la } \\
\text { Inclusión Socail condicionan } \\
\text { sus transferencias a la } \\
\text { asistencia escolar }\end{array}$ & $\begin{array}{l}\text { PISA (2000 y 2006), TIMSS } \\
\text { (1995 y 2003), PIRLS (2001), } \\
\text { Operativo Nacional de } \\
\text { Evaluación (2005) }\end{array}$ \\
\hline Brasil & $\begin{array}{l}\text { FUNDEF ha exigido } \\
\text { escolarización primaria } \\
\text { universal a municipios. Bolsa } \\
\text { Familia condiciona sus } \\
\text { transferencias a la } \\
\text { asistencia escolar }\end{array}$ & $\begin{array}{l}\text { PISA (2000, 2003, 2006), } \\
\text { Sistema de Avaliação da } \\
\text { Educação Bàsica (SAEB) }\end{array}$ \\
\hline Chile & $\begin{array}{l}\text { Chile Solidario condiciona sus } \\
\text { transferencias a la asistencia } \\
\text { escolar }\end{array}$ & $\begin{array}{l}\text { PISA (2000 y 2006), TIMSS } \\
\text { (1999 y 2003), International } \\
\text { Adult Literacy Survey, Sistema } \\
\text { de Medición de la Calidad } \\
\text { Educativa (SIMCE) }\end{array}$ \\
\hline \multicolumn{3}{|c|}{ Difusión } \\
\hline Argentina & \multicolumn{2}{|c|}{$\begin{array}{c}\text { Plan Social Educativo (focalización educativa técnica) } \\
\text { Plan Integral de Igualdad Educativa (focalización } \\
\text { educativa comunitaria) }\end{array}$} \\
\hline Brasil & \multicolumn{2}{|c|}{$\begin{array}{c}\text { Programa FUNDESCOLA } \\
\text { (focalización educativa técnica) }\end{array}$} \\
\hline Chile & \multicolumn{2}{|c|}{$\begin{array}{c}\text { Programa } 900 \text { Escuelas (focalización educativa } \\
\text { técnica, y luego comunitaria) } \\
\text { PROEIB, Escuela Rural, Liceo para Todos } \\
\text { (focalización educativa técnica) }\end{array}$} \\
\hline
\end{tabular}

Fuente: Elaboración propia.

Por otro lado, los tres gobiernos han recibido sugerencias para que articulen sus programas de protección social con el sistema educativo, tal como ya hemos visto que proponía el informe latinoamericano del Banco Mundial (WORLD BANK, 2004b). 
Esta idea ha inspirado las transferencias sociales condicionadas a la asistencia escolar efectiva de los hijos (también a su vacunación), que esperan imprimir un impulso a las comunidades más pobres además de acrecentar su capital humano.

La medición de los rendimientos académicos mediante exámenes estándar también ha arraigado. Argentina ha participado en muchos exámenes internacionales (PISA 2000 y 2006; TIMSS 1995 y 2003; PIRLS 2001), y llevó a cabo su Operativo Nacional de Evaluación en 2005. Brasil ha aplicado el examen PISA (2000, 2003, 2006) y ha implantado también el Sistema da Avaliação da Educação Básica (SAEB), que cruza los datos socioeconómicos del alumnado con su rendimiento en exámenes estándares. Chile ha generado y seguido los datos resultantes de PISA (2000 y 2006), TIMSS (1999 y 2003) e IALS. La creación del Sistema de Medición de la Calidad Educativa (SIMCE) fue una decisión de los gobiernos autoritarios asumida más adelante por los democráticos.

En definitiva, una parte sustancial de las medidas destinadas a la Educación para Todos responde a unas reglas políticas comunes a varios países. Ante todo, la influencia externa ha sido notoria en la incorporación de condiciones educativas a la protección social y en el uso de los exámenes internacionales. Por lo común esta protección social más atenta a la educación y aquella educación evaluada con exámenes no forman un dispositivo institucional congruente; cuando menos, en Brasil y en Chile han saltado a primer plano las ineficiencias e inequidades educativas, observadas por sendos sistemas de evaluación, pero no se vislumbran proyectos generales para superarlas (REIMERS et al., 2006; GARCIA-HUIDOBRO; BELLEÏ, 2006). Una posible explicación de este hecho podría recordar que la estandarización ha traído esta nueva regla de evaluación cuantitativa, pero no ha interpelado a los gobiernos sobre las estrategias, los pasos y los plazos de una política pública educativa consciente (DALE, 1999). Al fin y al cabo, ninguno de los tres ministerios de educación parece saber a ciencia cierta cómo sus medidas y programas pueden mejorar los datos de estos exámenes, tanto en comparación con otros países como con varios momentos del propio sistema educativo

Estos gobiernos también han incluido en su agenda la pugna por ampliar la educación de calidad a todos por medio de sendas estrategias de "focalización educativa". Este método de trabajo socio-educativo se inspira en una analogía de la protección social, ya que pretende concentrar los recursos escasos en los colectivos más necesitados, en este contexto unos recursos pedagógicos. Aun y el diferente arraigo de esta filosofía en los tres países, en todos ellos se han abierto vivas controversias políticas sobre el tema, que han teñido los programas y las propuestas de los colores ideológicos de los respectivos arcos políticos.

No obstante, el debate nacional se ha circunscrito a dos de lecturas políticas de la focalización (véase cuadro 3). Básicamente, la acepción técnica de este método compensatorio considera que los materiales, asesoramientos y estímulos pueden catalizar el cambio de las escuelas menos aventajadas, mientras que la acepción comunitaria añade que el reconocimiento profesional del profesorado y la participación familiar tienen tanta 
importancia como las medidas de apoyo técnico. En la polémica se han alineado tres grupos: los detractores conservadores rechazan las becas por vulnerabilidad en nombre de las becas por esfuerzo (FONTAINE TALAVERA, 2002); los partidarios de la focalización técnica sostienen que las eventuales limitaciones de los primeros experimentos se solventarían con mejoras de la formación docente y una evaluación más visible del aprendizaje (AGUERRONDO, 2005); y los partidarios de la focalización comunitaria hacen hincapié en la compensación y la implicación familiar en la escuela (GELDEREN, 2005). En Brasil también se ha abierto una disputa entre los defensores de una focalización mínima y de una focalización orientada por principios universalistas (SILVA, 2005), si bien los matices pedagógicos no han adquirido tanta importancia en este país como en sus vecinos.

Cuadro 3. Las versiones técnica y comunitaria de la focalización educativa

\begin{tabular}{|c|c|c|}
\hline $\begin{array}{l}\text { 'Función de producción } \\
\text { educativa' }\end{array}$ & $\begin{array}{l}\text { Focalización educativa } \\
\text { técnica }\end{array}$ & $\begin{array}{l}\text { Focalización educativa } \\
\text { comunitaria }\end{array}$ \\
\hline \multicolumn{3}{|c|}{ Intervención sobre el 'contexto' del sistema educativo } \\
\hline $\begin{array}{l}\text { Contrarrestar la segregación } \\
\text { escolar }\end{array}$ & \multicolumn{2}{|l|}{ No se discute } \\
\hline \multicolumn{3}{|c|}{ Intervención sobre los 'procesos' del sistema educativo } \\
\hline Pedagogía & \multicolumn{2}{|c|}{$\begin{array}{l}\text { Las pedagogías constructivistas ofrecen nuevas herramientas para } \\
\text { acercar la escuela a los sectores sociales pobres o excluidos }\end{array}$} \\
\hline Mejora de los hogares & \multicolumn{2}{|c|}{$\begin{array}{l}\text { La protección social debe ir acompañada de estímulos activa- } \\
\text { dores para inducir estrategias autónomas de promoción social a } \\
\text { los adultos, jóvenes y niños de los hogares pobres. Si las madres } \\
\text { pueden gestionar las ayudas, o su prestigio recibe un cierto } \\
\text { refuerzo por parte de los servicios sociales, el conjunto del hogar } \\
\text { experimenta una mejora todavía mayor. }\end{array}$} \\
\hline Mejora escolar & $\begin{array}{l}\text { Las escuelas necesitan materiales } \\
\text { pedagógicos, asesoramiento } \\
\text { profesional, mejoras infraes- } \\
\text { tructurales y estímulos a la } \\
\text { innovación pedagógica para } \\
\text { mejorar. Las escuelas cuyos } \\
\text { estudiantes concentran y } \\
\text { acumulan muchas desventajas } \\
\text { sociales incluso experimentan } \\
\text { una mayor necesidad. }\end{array}$ & $\begin{array}{l}\text { Además de todos los recursos } \\
\text { anteriores, el profesorado nece- } \\
\text { sita unas condiciones laborales } \\
\text { dignas y un cierto reconoci- } \\
\text { miento profesional para impul- } \\
\text { sar mejoras efectivas. Las familias } \\
\text { se revelan imprescindibles } \\
\text { cuando se abren los canales } \\
\text { participativos adecuados. Todo } \\
\text { ello es mucho más necesario en } \\
\text { los centros con desventajas con- } \\
\text { centradas y acumuladas. }\end{array}$ \\
\hline
\end{tabular}

Fuente: Elaboración propia a partir de las entrevistas en Argentina (2004, 2006, 2008), Brasil (2004, 2005) y Chile (2003). ${ }^{1}$ 
En resumen, la Educación para Todos pretende que "los servicios educativos funcionen para los pobres". Ello se puede conseguir asociando las ayudas sociales con la asistencia escolar, haciendo públicos los resultados académicos y compensando las desigualdades educativas. Estas líneas de actuación han llegado al Cono Sur a través de la estandarización y la difusión. Los sistemas normalizados de evaluación han abierto varios debates pero no han indicado los impactos precisos de las medidas adoptadas en ninguno de los tres países.

\section{LA COMERCIALIZACIÓN DE LOS SERVICIOS EDUCATIVOS}

El Acuerdo General de Comercio de los Servicios es un nodo político global donde convergen las principales vías supra-estatales de influencia en la política educativa y concurren todos los actores de la gobernanza mundial. En pocas palabras, el tratado ha estandarizado el contenido de los servicios educativos, y se ha inscrito en la difusión de varias creencias causales sobre las externalidades positivas del libre comercio.

El cuadro 4 resume el catálogo de servicios educativos según el AGCS, así como las principales barreras comerciales que el mismo acuerdo pretende derribar. De este modo regula la legislación de sus miembros, sus criterios de acreditación de operadores, de materiales didácticos o de organización de los mismos exámenes estandarizados (nacionales e internacionales). Al margen de la casuística, que no podemos detallar, cabe subrayar que en este punto se detecta una serie de reglas del juego que se instauran, de modo más o menos formal, con la misma participación en el acuerdo. Éstas esbozan el mismo concepto de servicio educativo, extensible desde la gestión de un centro hasta la administración de un examen, y podrían convertirse en una especie de profecía autosatisfactoria sobre el surgimiento de un mercado especializado en ellos a medio plazo.

En particular, es muy significativo el proyecto de remover las barreras a la presencia comercial. Si se cumpliesen estas condiciones del todo, fácilmente podría existir un mercado mundial en varios sectores educativos, donde unos mismos proveedores pudiesen vender sus programas educativos en todas partes, acreditándolos con las licencias nacionales, contratando el personal ajustado a sus previsiones y evitando métodos específicos de evaluación institucional.

La definición de la comercialización internacional de los servicios ha sido uno de los ejemplos históricos más claros de la influencia externa sobre las políticas gubernamentales por medio de la difusión. En un inicio ni los tratados ni los organismos internacionales contemplaban esta posibilidad, porque pensaban que este tipo de comercio internacional sería inviable. Pero luego un grupo académico consiguió convencer a varios gobiernos del valor de los servicios, los Estados Unidos empezaron a poner propuestas en este sentido sobre las mesas de negociación, y finalmente los defensores de la idea pudieron reprochar a los detractores que ocultaban intereses o creencias proteccionistas. 
Cuadro 4. Modos de comercio de servicios educativos, tipos de servicios y principales barreras

\begin{tabular}{|l|l|l|}
\hline Modo Comercial & $\begin{array}{l}\text { Servicios Educativos } \\
\text { (Ejemplos) }\end{array}$ & Principales Barreras (P.Ej.) \\
\hline 1.Suministro transfronterizo & $\begin{array}{l}\text { - Educación a distancia } \\
\text { - 'e-learning' } \\
\text { - 'Testing services' }\end{array}$ & $\begin{array}{l}\text { - Falta de oportunidad de } \\
\text { acreditación como } \\
\text { institución con capacidad de } \\
\text { otorgar títulos }\end{array}$ \\
\hline 2. Consumo en el extranjero & $\begin{array}{l}\text { Estudios en el extranjero } \\
\text { 3. Presencia comercial }\end{array}$ & $\begin{array}{l}\text { - Requerimientos de visado } \\
\text { Restricciones laborales } \\
\text { durante periodo de estudios }\end{array}$ \\
\hline $\begin{array}{l}\text { - Filiales de centros educativos } \\
\text { en el extranjero } \\
\text { Asociación entre centros } \\
\text { locales y extranjeros } \\
\text { Franquicias con } \\
\text { instituciones locales }\end{array}$ & $\begin{array}{l}\text { Se prohíbe a entidades } \\
\text { extranjeras proveer servicios } \\
\text { No acceso a licencia para } \\
\text { otorgar títulos reconocidos, } \\
\text { exámenes controlados por } \\
\text { colegios profesionales }\end{array}$ \\
\hline $\begin{array}{l}\text { Monopolios estatales } \\
\text { naturales }\end{array}$ & $\begin{array}{l}\text { Profesorado e investigadores } \\
\text { trabajando en el extranjero }\end{array}$ & $\begin{array}{l}\text { Requisitos de inmigración o } \\
\text { de residencia }\end{array}$ \\
\hline
\end{tabular}

Fuente: Elaboración con datos de GARCÍA-GUADILLA (2003), ROBERTSON et al. (2002) y VERGER (2006).

Si bien las primeras propuestas despertaron suspicacias en los años setenta, más adelante la U.S. Trade Representative (USTR) lanzó una fuerte campaña para introducir este tema en la agenda política internacional, a la que contribuyeron las principales empresas de servicios de los Estados Unidos (con un papel destacado de American Express), la OCDE y algunos laboratorios de ideas como el Trade Policy Research Center (CHANDA, 2003; MUNDY; IGA, 2003). De este modo, en la Ronda de Uruguay bastantes gobiernos empezaron a ser permeables a estos argumentos, especialmente las nuevas potencias como India o Brasil. A pesar de todo, continuaron negándose a tolerar que la inversión extranjera directa en la educación, y ratificaron el principio de que la liberalización del comercio de los servicios sólo era aceptable en la medida en que no dañase los objetivos de desarrollo del Sur (DRAKE; NICOLAIDIS, 1992). Pero acabaron aceptando un tratado global cuando los países del Norte flexibilizaron su postura sobre la agricultura y los servicios. Aunque hasta entonces nadie había mencionado la educación entre los servicios susceptibles de responder a un régimen 
comercial, acabó entrando en la lista subrepticiamente porque figuraba en el catálogo oficial de servicios adoptado, la Central Product Classification (CPC) de la División de Estadística de las Naciones Unidas. Todo esto ocurrió sin que la comunidad educativa reconociese su importancia, tal como muestra el hecho de que algún otro sector más informado -p.ej. la aeronáutica- consiguió su exención de los acuerdos (SCHERRER, 2005, p. 971).

Argentina, Brasil y Chile, pues, se encontraron en los años noventa con una incipiente comercialización de la educación superior en un contexto institucional favorable a esta posibilidad pero impreciso en sus términos. Hasta el momento, han adoptado cada uno su estrategia, que a grandes rasgos les permite gestionar precisamente estas ambigüedades. Así, en Argentina y en Brasil las propuestas liberalizadoras fueron bien recibidas en los años noventa, pero los ministros de educación de ambos países han cambiado estas percepciones más adelante firmando la Declaración de Brasilia, que precisamente reclama una cláusula que excluya la educación del AGCS. A pesar de todo, la estrategia efectiva del Ministerio de Comercio de Argentina apunta directamente en el sentido de la comercialización de los servicios educativos. Por su lado, el gobierno chileno expresa muchas menos reticencias, e incluso ha aceptado incluir la educación en algunos tratados de libre comercio.

A través de dos muestras de entrevistas llevadas a cabo en Argentina y Chile (véase nota 2), podemos perfilar el abanico de posiciones en la red de actores de las políticas educativas de estos países. A grandes rasgos, estas tomas de partido responden a varios motivos ideológicos, asumen un concepto de la educación y desembocan en la propensión de un agente a la liberalización comercial de los servicios educativos (vid. Cuadro 5). En general, tanto la oposición como la adhesión al librecambio educativo responden a principios morales, mientras que las posiciones intermedias se apoyan sobre varias 'creencias causales' acerca de los efectos hipotéticos de la apertura comercial en este sector.

Ciertamente, la oposición al acuerdo comercial recurre a una cierta hipótesis causal, ya que sobreentiende que la liberalización educativa puede ser devastadora, pero su primer motivo estriba en el principio de que no se puede mercantilizar un bien público. En cambio, las creencias fácticas proporcionan la guía que orienta a las posiciones de cautela, proteccionismo y oportunismo. Poniendo el concepto de la educación entre paréntesis, para estas perspectivas puede ser inconveniente añadir la educación a la lista de sectores sometidos al AGCS o a otros tratados comerciales, si éstos contienen imprecisiones sobre las actividades de referencia, o bien si el país carece de instituciones educativas competitivas internacionalmente en el sector que abre a los operadores extranjeros. Nótese que la propensión de estos actores políticos al librecambio educativo es mínima en las condiciones institucionales y estructurales actuales, pero su opinión es muy sensible a reformas políticas internacionales o a cambios en su economía nacional. Desde la perspectiva oportunista, la decisión de abrir la educación depende de un saldo de beneficios 
y costes de todos los servicios, con lo cual de hecho es coyuntural. Por último, en el extremo más proclive al librecambio de nuevo los principios se sobreponen a las teorías causales, o más bien éstas se subsumen en aquellos, puesto que las ventajas del librecambio educativo se convierten aquí en un axioma además de una oportunidad estratégica.

Cuadro 5. Posiciones políticas sobre el tratamiento de la educación en el AGCS

\begin{tabular}{|c|c|c|c|c|c|}
\hline & Oposición & Cautela & Proteccionismo & Oportunismo & Librecambio \\
\hline Motivos & $\begin{array}{l}\text { Principios } \\
\text { morales } \\
\text { (“la educación } \\
\text { no es una } \\
\text { mercancía”) }\end{array}$ & $\begin{array}{l}\text { Teorías causa- } \\
\text { efecto } \\
\text { (contenido del } \\
\text { AGCS) }\end{array}$ & $\begin{array}{l}\text { Teorías } \\
\text { causa-efecto } \\
\text { (peligros de la } \\
\text { liberalización) }\end{array}$ & $\begin{array}{l}\text { Motivos } \\
\text { instrumentales }\end{array}$ & $\begin{array}{l}\text { Oportunidades } \\
\text { de la } \\
\text { liberalización }\end{array}$ \\
\hline $\begin{array}{l}\text { Concepto de } \\
\text { la educación }\end{array}$ & $\begin{array}{l}\text { Bien público } \\
\text { proveído por } \\
\text { el estado }\end{array}$ & $\begin{array}{l}\text { El concepto de } \\
\text { la educación } \\
\text { no influye en } \\
\text { las decisiones } \\
\text { sobre el AGCS }\end{array}$ & $\begin{array}{l}\text { Prioridad a los } \\
\text { proveedores } \\
\text { nacionales }\end{array}$ & $\begin{array}{l}\text { La educación } \\
\text { es una moneda } \\
\text { de cambio }\end{array}$ & $\begin{array}{l}\text { La educación } \\
\text { debe ser una } \\
\text { mercancía } \\
\text { porque es un } \\
\text { bien escaso }\end{array}$ \\
\hline $\begin{array}{l}\text { Propensión a } \\
\text { liberalizar los } \\
\text { servicios } \\
\text { educativos }\end{array}$ & $\begin{array}{l}\text { Nula. La } \\
\text { liberalización } \\
\text { es innego- } \\
\text { ciable }\end{array}$ & $\begin{array}{l}\text { Mínima. Para } \\
\text { negociar este } \\
\text { punto es } \\
\text { imprescindible } \\
\text { que se aclaren } \\
\text { las zonas grises } \\
\text { del AGCS }\end{array}$ & $\begin{array}{l}\text { Mínima. Para } \\
\text { negociar el } \\
\text { AGCS es } \\
\text { imprescindible } \\
\text { contar con la } \\
\text { regulación } \\
\text { adecuada, o } \\
\text { bien con un } \\
\text { sector educa- } \\
\text { tivo nacional } \\
\text { competitivo }\end{array}$ & $\begin{array}{l}\text { Media. Es } \\
\text { aceptable } \\
\text { asumir } \\
\text { compromisos } \\
\text { de } \\
\text { liberalización } \\
\text { según los } \\
\text { resultados de } \\
\text { la negociación } \\
\text { en otras áreas }\end{array}$ & Elevada \\
\hline
\end{tabular}

Fuente: Elaboración propia a partir de las entrevistas en Argentina y Chile (2006).

Hemos anotado anteriormente que nuestro primer sondeo de las posiciones en Argentina, Brasil y Chile muestra algunas inconsistencias de los gobiernos en los dos primeros casos. En efecto, Argentina y Brasil han suscrito la Declaración de Brasilia para excluir la educación del AGCS, pero no tenemos evidencia concluyente de que hayan rechazado las oportunidades de liberalizar sus sectores educativos. Por su lado, los gobiernos chilenos han aceptado con muchas menos reticencias tratados comerciales que pueden afectar a la educación, pero el hecho de que la educación sea una de sus prioridades políticas, y la circunstancia de crisis al respecto en el país, ponen en duda que de momento se adhieran fácilmente a posiciones radicalmente favorables. Todo ello nos induce a la sospecha, exploratoria y tentativa, de que probablemente las creencias fácticas sobre los efectos de la apertura comercial educativa sean decisivas para fijar las posiciones de estos gobiernos a medio plazo. 


\section{CONCLUSIONES}

Uno de los síntomas más reveladores de la globalización es el surgimiento de nuevos canales por los que los actores políticos y los agentes sociales globales inciden en las decisiones políticas de los gobiernos. La educación es un ámbito de las políticas públicas en que este fenómeno es visible, especialmente en algunas regiones, como el Cono Sur, a las que los discursos recientes de los organismos internacionales aluden con frecuencia. En este artículo consignamos tres indicios sobre la vigencia de estas vías de influencia externa.

En primer lugar, las políticas educativas aplicadas en el Cono Sur se inspiran en unas mismas creencias fácticas sobre el desarrollo, similares a las tesis denominadas del Consenso de Washington. Aquellas teorías de hace veinte años concedían la primacía causal de la prosperidad material al funcionamiento irrestricto de los mercados, y esperaban de éste los avances propios del desarrollo social. En el programa EPT y el AGCS se desvela una sensibilidad extrema de los actores políticos nacionales a las creencias fácticas que circulan por estos medios globales.

En segundo lugar, los gobiernos del Cono Sur han aplicado el principio de la Educación para Todos con unas reglas internacionales normalizadas sobre el presupuesto educativo y la evaluación institucional, y han dado prioridad a unas medidas compensatorias encarecidamente recomendadas en los foros internacionales. Estas líneas de actuación han llegado al Cono Sur a través de la estandarización y la difusión. Se han estandarizado el gasto educativo, la vinculación de las transferencias sociales condicionadas a la escolaridad efectiva de los menores, y los sistemas de evaluación institucional mediante exámenes homogenizados. Al mismo tiempo, se ha difundido el método de la focalización o acción selectiva a favor de los colectivos menos afortunados. En el ámbito de la protección social adopta la forma de las ayudas condicionadas, como los ejemplos mencionados; en el ámbito educativo concentra en las escuelas desfavorecidas el asesoramiento pedagógico y, a veces, el esfuerzo por abrir canales de participación. No obstante, aunque los sistemas normalizados de evaluación han abierto varios debates de fondo sobre los avances y las fracturas educativas, no se ha concretado en ninguno de los casos cómo puede la focalización llegar a unos mejores resultados en estos sistemas de evaluación. Esta es otra muestra de la sensibilidad de las políticas nacionales a las influencias externas por la vía de las ideas globales sobre la educación, así como a la dificultad de cristalizar articulaciones nacionales congruentes de todas estas influencias.

Finalmente, el AGCS ha estandarizado un catálogo de servicios educativos. Además, sus promotores han difundido la idea de que el comercio internacional de estos servicios es una oportunidad económica para los países ricos y pobres. Los primeros encuentran nuevas oportunidades de exportación en un sector donde tienen garantizada la ventaja comparativa, pero los segundos esperan mejores aportaciones a su capital humano. Al menos, el Ministerio de Comercio argentino y el gobierno chileno confían notablemente en las creencias causales sobre las ventajas del libre comercio educativo. 


\section{Notas}

1. Las entrevistas fueron realizadas a políticos del área educativa y social, a personal técnico de los diversos programas focalizados y a profesorado de escuelas objeto de atención focalizada y/o con alumnado beneficiario de programas focalizados. En total se realizaron más de 50 entrevistas en los países donde se realizó el trabajo empírico.

\section{Referencias}

BONAL, X. Globalización y política educativa. Un análisis crítico de la agenda del Banco Mundial para América Latina. Revista Mexicana de Sociología, México, v. 64, p. 3-35, 2002.

CARNOY, M. Globalization and educational reform. Paris: Unesco- International Institute for Educational Planning, 1999.

CHANDA, R. Social Services and the GATS: Key Issues and Concerns. World Development, Montréal (Canada), v. 31, n. 12, p. 1.997-2.011, 2003.

DALE, R. Specifying Globalisation Effects on National Policy: focus on the Mechanisms. Journal of Education Policy, London (GB), v. 14, p. 1-17, 1999.

Globalisation and Education: Demonstrating a 'Common World Education Culture' or Locating a 'Globally Structured Educational Agenda'? Educational Theory, Urbana Champaign (USA), v. 50, p. 427-448, 2000.

Globalisation, knowledge economy and comparative education. Comparative Education, London (GB), v. 41, p. 117-149, 2005.

DRAKE, W. J.; NICOLAIDIS, K. Ideas, Interests, and Institutionalization: “Trade in Services" and the Uruguay Round. International Organization, Baltimore (USA), v. 46, n. 1, p. 37-100, 1992. FEKETEKUTY, G. International Trade in Services. An overview and blueprint for negotiations. Institute for Trade and Commercial Diplomacy. [on line]. Disponível em: <www.commercialdiplomacy.org>, 2005.

FONTAINE TALAVERA, A. Equidad y calidad en educación: cinco proposiciones interrelacionadas. Revista de Estudios Públicos, Santiago (Chile), v. 87, p. 5-38, 2002.

GARCÍA-GUADILLA, C. Balance de la década de los '90 y reflexiones sobre las nuevas fuerzas de cambio en la educación superior. In: MOLLIS, M. Las universidades en América Latina: ¿Reformadas o alteradas?. Buenos Aires: Clacso, 2003.

GARCÍA-HUIDOBRO, J. E.; BELLEÏ, C.¿Remedio para la inequidad? La subvención escolar preferencial. Revista Mensaje, Santiago (Chile), v. 55, n. 547, p. 40-44, mar./abr. 2006.

GELDEREN, A. M. van. Aportes y propuestas individuales y generales, sin diagnósticos. In: TEDESCO, J. C. ¿Cómo superar la desigualdad y la fragmentación del sistema educativo argentino?. Buenos Aires: Unesco- IIPE, p. 291-308, 2005.

GREEN, A. Education and globalization in Europe and East Asia: convergent and divergent trends. Journal of Education Policy, London (GB), v. 14, p. 55-71, 1999.

HAY, C. Political Analysis. London: Palgrave, 2002.

KELSEY, J. Legal Fetishism and the Contradictions of the GATS. Globalisation, Societies and Education, London (GB), v. 1, n. 3, p. 267-280, 2003. 
MUNDY, K.; IGA, M. Hegemonic Exceptionalism and Legitimating Bet-Hedging: paradoxes and lessons from the US and Japanese approaches to education services under the GATS. Globalisation, Societies and Education, London (GB), v. 1, n. 3, p. 321-357, 2003.

OZGA, J. S.; SEDDON, T.; POPKEWITZ, T. S. (Eds.). Education Research and Policy: Steering the Knowledge-based Economy. London: Routledge, 2006.

PAWSON, R. Evidence and Policy and Naming and Shaming. Policy Studies, London (GB), v. 23, n. 3-4, p. 211-230, sep./dec. 2002.

REIMERS F.; SHANO DA SILVA, C.; TREVINO, E. Where is the "education" in Conditional Cash Transfers in Education? Unesco Institute for Statistics Working Papers, Montréal (Canada), n. 4, 2006.

RIZVI, F; LINGARD, B. Globalization and the Changing Nature of the OECD's Educational Work. In: LAUDER, H.; BROWN, Ph.; DILlABOUG, J. A.; HALSEY, A. H. Education, Globalization and Social Change. Oxford: Oxford University Press, 2006.

ROBERTSON, S.; BONAL, X.; DALE, R. GATS and the Education Service Industry: The Politics of Scale and Global Reterritorialization. Comparative Education Review, Chicago (USA), v. 46, p. 472-496, 2002.

ROBERTSON, S.; DALE, R. This is what the fuss is about! The implications of GATS for education systems in the north and the south. Presented at UK Forum for International Education and Training, London, 2003.

ROBERTSON, S.; NOVELLI, M.; DALE, R.; TIKLY, L.; DACHI, H.; NDEBELA, A. Education and Development in a Global Era (Vol. II): ideas, actors and dynamics in the global governance of education. London: Department for International Development, 2007.

SAUVÉ, P. Trade, Education and the GATS: What's In, What's Out, What's All the Fuss About. Paris: OECD- Ceri, 2002.

SCHERRER, C. GATS: long-term strategy for the commodification of education. Review of International Political Economy, Baltimore (USA), v. 12, n. 3, p. 484-510, 2005.

SILVA, M. O. From a Minimum Income to a Citizenship Income: the Brazilian Experiences. Institut de Drets humans de Catalunya, Barcelona (España), v. 1, 2005.

TARABINI, A. The spread of targeted educational policies in Latin America: global thesis and local impacts. International Studies in Sociology of Education, London (GB), v. 17, p. 21-43, 2007.

UNESCO. EFA Global Monitoring Report. The Quality Imperative. Paris: Unesco, 2005.

UNESCO- LLECE (Laboratorio Latinoamericano de Evaluación de la Calidad de la Educación). Estudio cualitativo de escuelas con resultados destacables en siete paises latinoamericanos. Santiago de Chile: Orealc- Unesco, 2002.

VERGER, A. La liberalización educativa en el marco del AGCS/GATS. Analizando el estado actual de las negociaciones. Archivos Analíticos de Politicas Educativas, Tempe (USA), v. 14, p. 1-23, 2006.

GATS and the global politics of higher education. London and New York: Routledge, 2009.

WORLD BANK. World Development Report: Making Services Work for Poor People. Washington: The World Bank Group, 2004a.

Group, 2004b.

Inequalities in Latin America: Breaking with the Past?. Washington: The World Bank 


\section{A influência externa nas políticas educativas da Argentina, Brasil e Chile (1990-2006) \\ Resumo}

O objetivo deste artigo é analisar o papel da influência internacional no delineamento e aplicação de politicas educativas no Cone Sul. Pretende explorar especialmente o processo através do qual as idéias, recomendaçôes e metas globais modelam as politicas educativas latino-americanas. Para isso o artigo centra-se no Programa de Educação para Todos e no Acordo Geral de Comércio de Serviço, analisando os mecanismos de influência externa que intervêm em cada caso-imposição, difusão e padronização-, as formas através das quais se "negociam" em niveis nacionais tais programas e acordos e os efeitos desses processos nas políticas educativas nacionais de três países da regiāo-Argentina, Brasil e Chile.

Palavras-chave: Globalização. Agenda Educativa Global. Educação para todos.

\section{The external influence in the educational policies of Argentina, Brazil and Chile (1990-2006)}

Abstract

The objective of the article is to analyse the role of international influence in the design and application of educational policies in the Southern Cone. Specifically it aims to explore the process by which the global ideas, recommendations and goals shape the Latin-American educational policies. Within this objective, the article is focused on the Education for All Programme and on the General Agreement on Trade and Services, analysing the mechanisms of external influence which intervene in each case imposition, dissemination and standardisation -, the ways by which these Programmes and Agreements are "negotiated" in national contexts, and the effects of these processes on national educational policies of three Latin-American countries - Argentina, Brazil and Chile.

Keywords: Globalisation. Global educational agenda. Education for All. GATS.

\section{L'influence externe dans les politiques éducationnelles de l'Argentine, le Brésil et le Chili (1990-2006) Résumé}

L'objectif de cet article est d'analyser le rôle de l'influence internationale dans le dessein et l'application de politiques éducationnelles dans le Cone Sud. Spécifiquement, il vise explorer le processus par lequel les idées, les recommandations et buts globaux donnent forme aux politiques éducationnelles de l'Amérique Latine. Suivant cet objectif, l'article est centré sur le Programme Éducation pour Tous et sur l'Accord Général sur les Services, analysant les mécanismes de l'influence externe qui interviennent dans chaque cas - imposition, dissémination et standardisation -, les moyens para lesquels ces Programmes et Accords sont 'négociés' dans les contextes nationaux et les effets de ces processus sur les politiques éducationnelles nationales de trois pays de l'Amérique Latine - Argentine, Brésil et Chili.

Mots clefs : Globalisation. Agenda Éducationnelle Globale. Éducation pour Tous. GATS. 
